\title{
External Tonehole Interactions in Woodwind Instruments
}

\author{
Antoine Lefebvre and Gary P. Scavone \\ Computational Acoustic Modeling Laboratory, \\ Centre for Interdisciplinary Research in Music Media and Technology (CIRMMT) \\ Schulich School of Music, McGill University, \\ 555 Sherbrooke Street West, Montréal, Québec H3A 1E3, Canada
}

\author{
Jean Kergomard \\ LMA, CNRS, UPR 7051, Aix-Marseille Univ, Centrale Marseille, \\ F-13402 Marseille Cedex 20, France
}

October 29, 2018

\begin{abstract}
The classical Transfer-Matrix Method (TMM) is often used to calculate the input impedance of woodwind instruments. However, the TMM ignores the possible influence of the radiated sound from toneholes on other open holes. In this paper a method is proposed to account for external tonehole interactions. We describe the Transfer-Matrix Method with external Interaction (TMMI) and then compare results using this approach with the Finite Element Method (FEM) and TMM, as well as with experimental data. It is found that the external tonehole interactions increase the amount of radiated energy, reduce slightly the lower resonance frequencies, and modify significantly the response near and above the tonehole lattice cutoff frequency. In an appendix, a simple perturbation of the TMM to account for external interactions is investigated, though it is found to be inadequate at low frequencies and for holes spaced far apart.
\end{abstract}

PACS: $43.75 \mathrm{Ef}, 4320 \mathrm{Rz}$

Keywords: radiation, musical instruments, reed instruments, clarinet

\section{Introduction}

A method to accurately and efficiently estimate the input impedance and resonance frequencies of woodwind instruments is of primary importance. The Transfer-Matrix Method (TMM) is typically used for this purpose (see e.g. Plitnik and Strong [25], Caussé et al. [3], Keefe [14]), because of its simplicity and efficiency, and it is the basis of software used by some instrument makers, such as RESONANS or BIAS. This method ignores internal interactions due to the coupling between the evanescent modes of nearby discontinuities as well as external interactions, which exists because the radiation impedance of each open tonehole is influenced by the radiation of sound from other toneholes. The problem of the response of woodwind instruments with external tonehole interactions was stated in a complete form by Leppington [21] and a method of solution was proposed by Kergomard [16] using the mutual radiation impedance proposed by Pritchard [26]. This method is based on the TMM for internal propagation with modified open tonehole radiation impedances to account for interactions (referred to as 
TMMI). Preliminary experimental results were obtained by Springer [27] for the case of holes spaced far apart in a pipe. No other validation of the method has been proposed since reference [16].

In this paper, we investigate the effect of external tonehole interactions in woodwind instruments with the TMMI. The first goal is to determine the validity of the method by comparing the results of TMMI calculations with Finite Element Method (FEM) simulations and wth measurements. We also compare these results with TMM calculations to show that some of the discrepancies are explained by the external interactions.

The second goal is to apply the proposed TMMI method to the case of woodwind instruments in order to determine the importance of the effect on their acoustical properties and to judge whether or not it is necessary to account for those interactions when calculating the input impedance of woodwind instruments for design purposes.

The theory of the TMM and TMMI, as well as the details of the FEM, are reviewed in the next section, and the presentation of the TMMI is completed in Appendix A for a general model of open holes. This is followed by the presentation of the results for a tube with a regular array of holes (Sec. 3), then results for a saxophone and a clarinet (Sec. 4) and finally the conclusions (Sec.5). In Appendix B the possibility to use a perturbation approach for the TMM is investigated.

\section{Background}

\subsection{The TMM}

The transfer matrix method (TMM) provides an efficient means for calculating the input impedance of a hypothetical air column [25, 3, 14]. With the TMM, a geometrical structure is approximated by a sequence of one-dimensional segments, such as cylinders, cones, and closed or open toneholes, and each segment is represented by a transfer matrix (TM) that relates its input to output frequency-domain quantities of pressure $(P)$ and volume velocity $(U)$. The multiplication of these matrices yields a single matrix which must then be multiplied by an appropriate radiation impedance, $Z_{\text {rad }}$, at its output as:

$$
\left[\begin{array}{c}
P_{\text {in }} \\
U_{\text {in }}
\end{array}\right]=\left(\prod_{i=1}^{n} \mathbf{T}_{i}\right)\left[\begin{array}{c}
Z_{\text {rad }} U_{\text {out }} \\
U_{\text {out }}
\end{array}\right] .
$$

The input impedance is then calculated as $Z_{\text {in }}=P_{\text {in }} / U_{\text {in }}$, without need to know $U_{\text {out }}$.

The theoretical expression of the transfer matrix of a cylinder is:

$$
\mathbf{T}_{c y l}=\left[\begin{array}{cc}
\cosh (\Gamma L) & Z_{0} \sinh (\Gamma L) \\
Z_{0}^{-1} \sinh (\Gamma L) & \cosh (\Gamma L)
\end{array}\right],
$$

where $Z_{0}=\rho c / \pi a^{2}, \Gamma=j \omega / c+(1+j) \alpha$ is the complex propagation constant, $\rho$ is the air density, $c$ the speed of sound, and $\omega$ the angular frequency. Losses are represented by $\alpha$, which depends on the radius $a$ of the cylindrical pipe and varies with the 
square root of the lossless wavenumber $k=\omega / c$ :

$$
\alpha=(C S T / a) \sqrt{k}
$$

where $C S T$ is a constant that depends of the properties of air:

$$
C S T=\sqrt{\ell_{v} / 2}(1+(\gamma-1) / \sqrt{\operatorname{Pr}})
$$

$\ell_{v}=\mu / \rho c$ is the characteristic length of viscous effects, $\mu$ is the dynamic viscosity, Pr the Prandtl number and $\gamma$ the ratio of specific heats. The formulas above for $Z_{0}$ and $\Gamma$ are sufficiently accurate for the present study.

The transfer matrix of a conical waveguide is (see Chaigne and Kergomard [4]):

$$
\mathbf{T}_{\text {cone }}=\left[\begin{array}{cc}
\left(a_{2} / a_{1}\right) \cos \left(k_{c} L\right)-\sin \left(k_{c} L\right) / k x_{1} & j Z_{c} \sin \left(k_{c} L\right) \\
Z_{c}^{-1}\left[j\left(1+\left(k^{2} x_{1} x_{2}\right)^{-1}\right) \sin \left(k_{c} L\right)+\left(x_{1}^{-1}-x_{2}^{-1}\right) \cos \left(k_{c} L\right) / j k\right] & \left(a_{1} / a_{2}\right) \cos \left(k_{c} L\right)+\sin \left(k_{c} L\right) / k x_{2}
\end{array}\right],
$$

where $a_{1}$ and $a_{2}$ are the radii at the input and output planes, respectively, and $x_{1}$ and $x_{2}$ are the distances between the apex of the cone and the input and output planes, $Z_{c}=\rho c /\left(\pi a_{1} a_{2}\right)$ and $k_{c}=-j \Gamma$ is the complex wavenumber. In this case, losses are evaluated at the equivalent radius [4]:

$$
a_{e q}=L \frac{a_{1}}{x_{1}} \frac{1}{\ln \left(1+L / x_{1}\right)} .
$$

The transfer matrix of a tonehole is defined as:

$$
\mathbf{T}_{\text {hole }}=\left(\begin{array}{cc}
1 & Z_{a} / 2 \\
0 & 1
\end{array}\right)\left(\begin{array}{cc}
1 & 0 \\
Z_{s}^{-1} & 1
\end{array}\right)\left(\begin{array}{cc}
1 & Z_{a} / 2 \\
0 & 1
\end{array}\right)
$$

where $Z_{a}$ is the series impedance and $Z_{s}$ the shunt impedance. These impedances have different values in the open and closed state. The calculation of these impedances has been the subject of many articles (Nederveen et al. [24], Dubos et al. [9], Dalmont et al. [7], Lefebvre and Scavone [20]) and the reader is referred to those papers for the appropriate formulas.

\subsection{The TMMI}

\subsubsection{Structure of the computation}

The radiation impedance of each tonehole on a woodwind instrument is influenced by the sound radiated from other holes. A method of solution to account for such interactions was proposed by Kergomard [16]. It can be used for any bore shape by making use of the classical TMM with modifications for the matrices located between open toneholes. It gives identical results 
to the TMM if interactions are neglected (by specifying null mutual radiation impedances). That is, the geometry is discretized identically, with both closed tonehole and open tonehole series impedance terms $Z_{a}$ represented as in the TMM.

We assume an instrument with $N$ openings (embouchure hole, toneholes, open end), where the indices of the openings range from $n=1$ to $N$. The pressure $P_{n}^{r a d}$ at opening $n$ is related to the acoustic flow $U_{n}^{\text {rad }}$ radiating out of hole $n$ by the following matrix relationship:

$$
\mathbf{P}^{r a d}=\mathbb{Z} \mathbf{U}^{r a d},
$$

where we define the vector $\mathbf{P}^{\text {rad }}$ of the pressures $P_{n}^{r a d}$ and the vector $\mathbf{U}^{r a d}$ of the flow rates $U_{n}^{r a d} . \mathbb{Z}$ is the radiation impedance matrix, which includes the effect of external interactions. The precise values of the different elements are difficult to determine. The self radiation impedances are the diagonal elements. The validity of this expression comes directly from the integral form of the Helmholtz equation if the Green function is chosen to satisfy the Neumann boundary conditions on the tube (see e.g. Eq. (7.1.17) in Morse and Ingard [23], see also Leppington [21]). As a consequence, the equations used by Keefe [13] are erroneous (see Eqs. A1a to A2b). The content of this matrix is explained in Sec.2.2.3.

A complete description of the planar mode propagation inside the tonehole chimney is possible, as explained in Appendix A. If the height is smaller than the wavelength, this can be simplified as:

$$
\begin{aligned}
P_{n} & =P_{n}^{\text {rad }}+B_{n} U_{n}, \\
U_{n}^{\text {rad }} & =U_{n},
\end{aligned}
$$

where $P_{n}$ is the pressure at the hole inside the air column and $B_{n}$ is the impedance of the total acoustic mass of the hole (see Appendix A). This approximation is possible for the frequency range of the present study. Using the diagonal matrix $\mathbb{B}$, we write:

$$
\mathbf{P}=\mathbf{P}^{\mathrm{rad}}+\mathbb{B} \mathbf{U}=(\mathbb{Z}+\mathbb{B}) \mathbf{U}
$$

An alternative equation relating the pressures and flows due to propagation inside the instrument can be derived for each hole $n$. As illustrated in Fig. 1, the sum of the flow $U_{n}$ radiating out of the tonehole, the flow $U_{n}^{\text {right }}$ entering the tonehole section on the right, and the flow $U_{n}^{\text {left }}$ entering the tonehole section on the left is equal to the flow source $U_{n}^{s}$ (which is discussed hereafter). This flow conservation equation can be written as:

$$
U_{n}^{s}=U_{n}+U_{n}^{\text {left }}+U_{n}^{\text {right }},
$$

where we note that the flow on the left is defined in the reverse direction. 


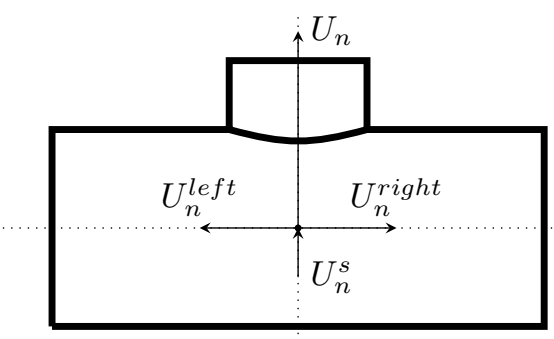

Figure 1: Diagram of the flow contributions in Eq. (11).

This equation can be written in a matrix form as:

$$
\mathbf{U}^{s}=\mathbf{U}+\mathbf{U}^{l e f t}+\mathbf{U}^{r i g h t}
$$

where $\mathbf{U}^{s}$ is the flow source vector. The sum of the left and right internal flows for each section is related to the pressures as

$$
\mathbf{U}^{l e f t}+\mathbf{U}^{\text {right }}=\mathbb{Y} \mathbf{P}
$$

where $\mathbb{Y}$ is the admittance matrix, which is described in Sec. 2.2.4

By combining Eqs. (10), 12) and (13, we obtain the solution:

$$
\mathbf{U}=[\mathbb{I}+\mathbb{Y}(\mathbb{Z}+\mathbb{B})]^{-1} \mathbf{U}^{s}
$$

where $\mathbb{I}$ is the identity matrix.

\subsubsection{Source Vector of Flow Rates $\mathbf{U}^{s}$}

For such a calculation, the flow-source vector $\mathbf{U}^{s}$ needs to be known. Generally speaking, the reader can imagine a small loudspeaker located inside the pipe at the abscissa of each hole, providing a flow rate $U_{n}^{s}$. Clearly, the resonator of a musical instrument is passive and such sources do not exist. All transfer functions between two acoustic quantities at every point in the space of the passive system are fully determined and the unique problem is the choice of a reference. A solution is to use as a reference the flow rate on the left at the first open tonehole of the instrument (from the part of the instrument that does not have any open holes), $-U_{1}^{l e f t}$. This quantity can be regarded as a source, if a source is defined as a fixed (or forced) quantity (in the sense of the Thevenin theorem). In the absence of an active source, Eq. (12) for this hole becomes:.

$$
-U_{1}^{l e f t}=U_{1}+U_{1}^{r i g h t}
$$


Thus, we can replace Eq. (12) for the first open hole by the following:

$$
U_{1}^{s}=U_{1}+U_{1}^{\text {right }}\left(=-U_{1}^{\text {left }}\right)
$$

Otherwise $U_{n}^{s}=0$ for $n \neq 1$. In this way, we can compute all quantities with respect to $U_{1}^{s}$, i.e. the ratio of all quantities to $U_{1}^{s}$. The input impedance can be easily deduced from the knowledge of $U_{1}^{s}$ and $P_{1}$, the ratio $U_{1}^{s} / P_{1}$ being the input admittance $Y^{u p}$ of the part of the system with open toneholes. Therefore the input impedance is classically computed by projecting the impedance $1 / Y^{u p}$ at the input of the instrument, or by using a transfer matrix relationship. Then all quantities can be calculated with respect to the input flow rate $U_{0}^{\text {right }}$ if necessary, where the index 0 refers to quantities at the input plane of the system. For reed instruments, this quantity is related to the input pressure by a time-domain nonlinear characteristic 1

\subsubsection{Radiation Impedance Matrix $\mathbb{Z}$}

The self-radiation impedances $Z_{n n}$ are approximately known (see e.g. Dalmont and Nederveen [6]). On the other hand, at low frequencies, the mutual radiation impedance $Z_{n m}$ (when $n \neq m$ ) is, assuming that holes radiate as monopoles (see Pritchard [26, Eq. (17)]):

$$
Z_{n m}=j k \rho c \frac{e^{-j k d_{n m}}}{2 \pi d_{n m}}
$$

where $d_{n m}$ is the distance between toneholes $n$ and $m$. More closely spaced toneholes have a larger mutual radiation impedance. As the mutual impedance is complex, both reactive and dissipative effects are expected. The factor 2 in the denominator corresponds to the radiation of a monopole into a half space. At very low frequencies a factor 4 would be more logical because the radiation is into a complete space, but empirically we noted that at higher frequencies, when the effect of interaction is especially important, a factor 2 is more suitable. It is difficult to determine the best approximation for the radiation impedance (see e.g. Dalmont and Nederveen [6], Dalmont et al. [7]).

Therefore, the impedance matrix $\mathbb{Z}$ is a full matrix. The mutual impedance may be neglected by using a diagonal matrix $\mathbb{D}$ with self impedance only, in which case the results are identical to those of the TMM.

\subsubsection{Admittance Matrix $\mathbb{Y}$}

The propagation of planar sound waves between two toneholes 1 and 2 can be described by classical transfer matrices:

$$
\left[\begin{array}{c}
P_{n} \\
U_{n}^{\text {right }}
\end{array}\right]=\left[\begin{array}{cc}
A_{n} & B_{n} \\
C_{n} & D_{n}
\end{array}\right]\left[\begin{array}{c}
P_{n+1} \\
-U_{n+1}^{\text {left }}
\end{array}\right],
$$

\footnotetext{
${ }^{1}$ For flute-like instruments, it should be possible to choose the flow rate $U_{1}$ exiting from the mouthpiece, which is the first open hole, as a source. However a complete nonlinear model needs to consider a pressure-difference source (i.e. a force source) near the edge, and it is necessary to add an equation in order to compute the flow rates radiating from the holes with respect to this source.
} 
where the transfer matrix is the multiplication of the transfer matrices of each segment located between the two open toneholes, including any closed toneholes. As explained above, the series impedances $Z_{a}$ of the open toneholes can be accounted for by including them in the transfer matrix, one-half on each side.

This matrix can be written in the form of an admittance matrix:

$$
\left[\begin{array}{c}
U_{n}^{\text {right }} \\
U_{n+1}^{\text {left }}
\end{array}\right]=\left[\begin{array}{cc}
Y_{n} & Y_{\mu, n} \\
Y_{\mu, n} & Y_{n}^{\prime}
\end{array}\right]\left[\begin{array}{c}
P_{n} \\
P_{n+1}
\end{array}\right],
$$

The parameters of this matrix are related to those of the transfer matrix: $Y_{n}=D_{n} / B_{n}, Y_{n}^{\prime}=A_{n} / B_{n}$ and $Y_{\mu, n}=-1 / B_{n}$, which assumes that $A_{n} D_{n}-B_{n} C_{n}=1$, the condition for reciprocity. The right and left flows at one tonehole section $n$ become:

$$
U_{n}^{\text {right }}=Y_{n} P_{n}+Y_{\mu, n} P_{n+1}
$$

and

$$
U_{n}^{\text {left }}=Y_{\mu, n-1} P_{n-1}+Y_{n-1}^{\prime} P_{n}
$$

Thus, Eq. (11) can be expanded to:

$$
U_{n}^{s}=U_{n}+Y_{\mu, n-1} P_{n-1}+\left(Y_{n-1}^{\prime}+Y_{n}\right) P_{n}+Y_{\mu, n} P_{n+1} .
$$

The coefficients of this equation define the admittance matrix $\mathbb{Y}$, which is tridiagonal. The first and last equations have to be modified because there is either no previous opening or no next opening. The last opening is located at the far end of the instrument, so that $U_{N}^{\text {right }}=0$ and Eq. (21) becomes simply:

$$
U_{N}^{s}=U_{N}+Y_{\mu, N-1} P_{N-1}+Y_{N-1}^{\prime} P_{N},
$$

where $U_{N}$ is the flow rate radiated at the end of the tube.

For the first opening, we use Eq. 15 , where we can set $U_{1}^{s}$, the first entry of the flow source vector, to any value. Then, using Eq. (14), solving the problem gives the flow vector $\mathbf{U}$. The pressure vector $\mathbf{P}$ can be deduced with Eq. (8).

\subsection{Finite Element Calculations}

The evaluation of the input impedance of woodwind instruments using the FEM involves constructing a 3D model of the air column surrounded by a radiation sphere and the solution of the Helmholtz equation for a number of selected frequencies. The body of the instrument itself is considered to be rigid. The mesh occupies the volume inside and outside the instrument. Curved third-order Lagrange elements are used. 
The input impedance (or reflectance) is evaluated from the FEM solution by evaluating the relationship of pressure and volume flow (or traveling-wave components of pressure) at the input plane of the system (see also Lefebvre and Scavone [20]). The surrounding spherical radiation domain uses a second-order non-reflecting spherical boundary condition on its surface, as described by Bayliss et al. [1]. Further discussion on this topic can be found in Tsynkov [28] and Givoli and Neta [10].

Thermoviscous boundary layer losses may be approximated with a special boundary condition such as presented by Cremer [5] and, more recently, Bossart et al. [2] or Kampinga et al. [12]. The boundary condition can be written as a specific acoustic admittance:

$$
Y_{w a l l}=-\frac{v_{n}}{p}=\frac{1}{\rho c} \sqrt{j k \ell_{v}}\left[\sin ^{2} \theta+(\gamma-1) / \sqrt{\operatorname{Pr}}\right]
$$

where $v_{n}$ is the normal velocity on the boundary and $\theta$ is the angle of incidence of the plane wave. The angle of incidence may be calculated from $\cos \theta=\hat{n} \cdot \hat{v} /\|\hat{v}\|$, where the normal vector $\hat{n}$ is of unit length. This is solved iteratively. The lossless problem is solved first, then the admittance on the boundary is calculated from the normal velocity of the solution and the problem is solved again. This is repeated until convergence is found.

The properties of air at $25^{\circ} \mathrm{C}$ are used for all the simulation cases. See Caussé et al. [3] for the equations used to calculate those values.

The reflectance $R(f)=p_{-} / p_{+}$(ratio of the reflected to incident pressure) is obtained from the simulation results. A cylindrical segment is added before the input plane of the object under study. The pressures $p_{a}$ and $p_{b}$ at two points on the centerline of this cylindrical segment, at distances $a$ and $b$ from the input plane, are extracted and the reflectance is calculated as:

$$
R=\frac{e^{-\Gamma b}-H_{b a} e^{-\Gamma a}}{H_{b a} e^{\Gamma a}-e^{\Gamma b}}
$$

where $H_{b a}=p_{b} / p_{a}$ is the transfer function between the two pressures and $\Gamma$ is as previously defined. A singularity in this equation exists when the distance is half of the wavelength. The reduced impedance can then be calculated with $\bar{Z}=$ $(1+R) /(1-R)$.

This method to calculate the reflectance was inspired by the two-microphones transfer function method of impedance measurement. It is worth mentioning that the impedance could also be calculated as $\overline{Z_{i n}}=p_{i n} / \rho c v_{i n}$, where the pressure and velocity are extracted directly at the input plane. When validating this approach using a cylindrical pipe, it was found that the results did not match theory as well as with the two-point method [18].

\section{Results for a Pipe with a Regular Array of Holes}

External tonehole interactions were studied experimentally by Springer [27] during an internship in Le Mans, France. The experiment involved measuring the internal pressure at the position of the holes on a tube with an array of widely spaced toneholes. The distances between the toneholes was much larger than what is found on woodwind instruments but the conclusion 


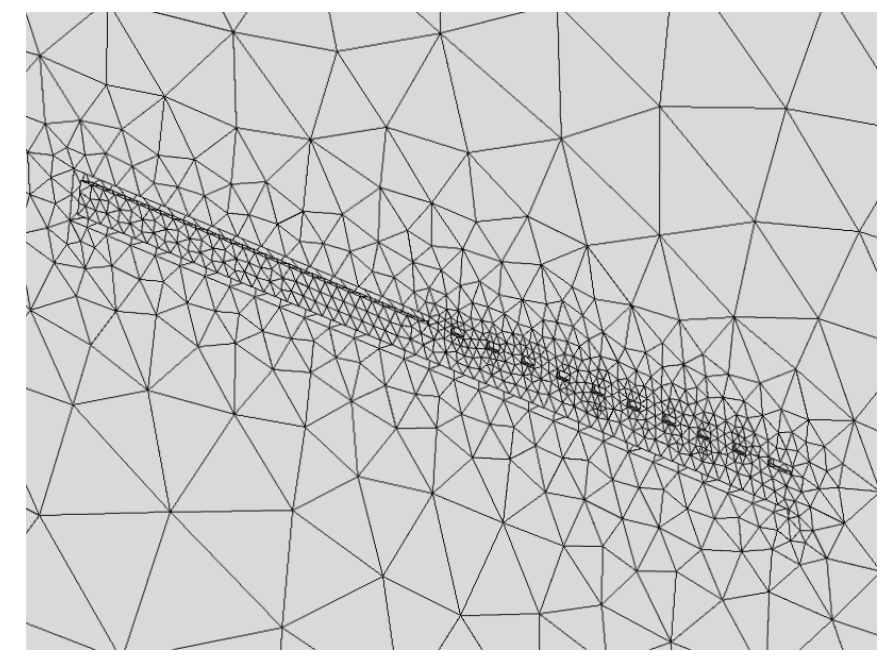

Figure 2: The mesh of a pipe with 10 open toneholes.

remains applicable to some extent. A cylindrical pipe of 4 meters length with an internal diameter of $15.3 \mathrm{~mm}$ was drilled with 47 holes of $8.7 \mathrm{~mm}$ diameter regularly separated by $8 \mathrm{~cm}$. The far end of the pipe was rigidly capped. The wall thickness was $3 \mathrm{~mm}$, and the temperature $20^{\circ} \mathrm{C}$. The excitation (white noise signal) was provided by a loudspeaker at the input of the tube. The internal pressure was measured at the positions of hole 1, 3 and 11 using 1/4-in B\&K microphones mounted flush with the pipe wall opposite the toneholes. The transfer functions with respect to the pressure at the first tonehole were calculated using an HP analyser and a computer. The results are shown in Figs. 3 and 4 in comparison to theoretical calculations with and without interactions.

For frequencies lower than the cutoff frequency of a tonehole lattice, the sound is exponentially attenuated inside the waveguide when interactions are ignored, whereas the external pressure is inversely proportional to distance. Therefore, the acoustic pressure coming from outside of the toneholes located farther down an instrument becomes stronger than the pressure coming from inside the instrument. In Fig. 3, it appears that the effect of the external interactions is negligible for the 3rd tonehole because the pressure coming from inside remains important, but in Fig. 4t the internal pressure has sufficiently decayed at the 11th hole such that the external sound field dominates. The phase curve is very instructive: when interactions are ignored the phase shift is very small, indicating evanescent waves, while when interactions are taken into account, the phase variation is linear, indicating (spherical) traveling waves.

Therefore the effect of interaction is extremely strong for this case (widely spaced holes and low frequencies). This is the reason why a perturbation method starting from the TMM cannot be used: this idea is investigated in Appendix B. Unfortunately, the convergence is limited to pass bands (above the first cutoff), and this method, used by Nederveen et al. [24, p.115], cannot be used for stop bands.

For frequencies higher than the cutoff frequency of the tonehole lattice, the internal pressure is no longer exponentially attenuated and the effect of interactions is limited to a smoothing of the response. 

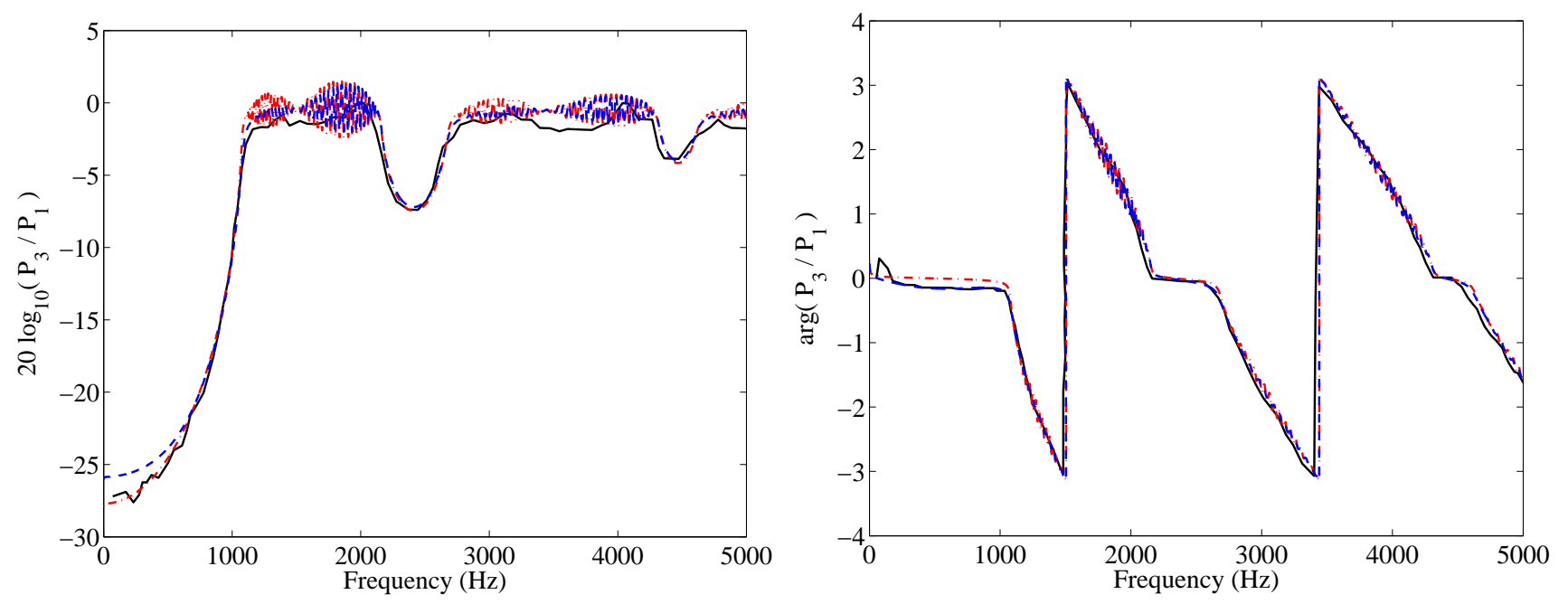

Figure 3: Modulus and argument of the transfer function between the internal pressure at hole 3 and 1: experimental results (black solid), theoretical calculation without external interactions (TMM, red dash-dotted) and with interactions (TMMI, blue dashed).

In order to better understand the impact of the existence of external interactions on the playing characteristics of woodwind instruments, the influence of the external interactions on the input impedance (or reflectance) of a pipe with an array of closely spaced toneholes was studied. The pipe was $303 \mathrm{~mm}$ in length, with a $12.7 \mathrm{~mm}$ diameter and $3.2 \mathrm{~mm}$ wall thickness. It was drilled with 10 holes of $9.5 \mathrm{~mm}$ diameter equally spaced by $15 \mathrm{~mm}$, starting at a position of $153 \mathrm{~mm}$ from the input end. The reflectance of that pipe was obtained with the proposed calculation method and compared to simulation results with the FEM, to experimental measurements (see description in the next section) and to calculations with the classical TMM. The magnitude of the reflectance and the equivalent length are plotted in Fig. 5.

An important observation is that the FEM results closely match the experimental measurement. This significantly increases our confidence in both the FEM and the experiment. For the equivalent length, the measurement error appears to be larger, particularly for the lower frequencies (below $1000 \mathrm{~Hz}$ ). There also seems to be a slight systematic error of a few millimeters. The proposed TMMI calculation to account for external interactions clearly gives better results than the TMM. The deep minima in the magnitude of the reflectance and the large increase in equivalent length in the higher frequencies completely disappear when interactions are included. The overall shape of the curves resemble the measured and simulated ones, even though some discrepancies remain. In the lower frequencies, the magnitude of the reflectance is reduced by the external interactions, which indicates a higher radiation efficiency. In the higher frequencies, the minima in the magnitude of the reflectance is not as low as in the measurement and is not located exactly at the same frequency. Small discrepancies also exist in the equivalent length, though they appear to be on the order of the measurement errors. In the lower frequencies, the external interactions increase the equivalent length slightly compared to predictions of the TMM. The toneholes on the pipe are located very close to each other, so that the evanescent modes excited near each discontinuity interact with those of adjacent toneholes, 

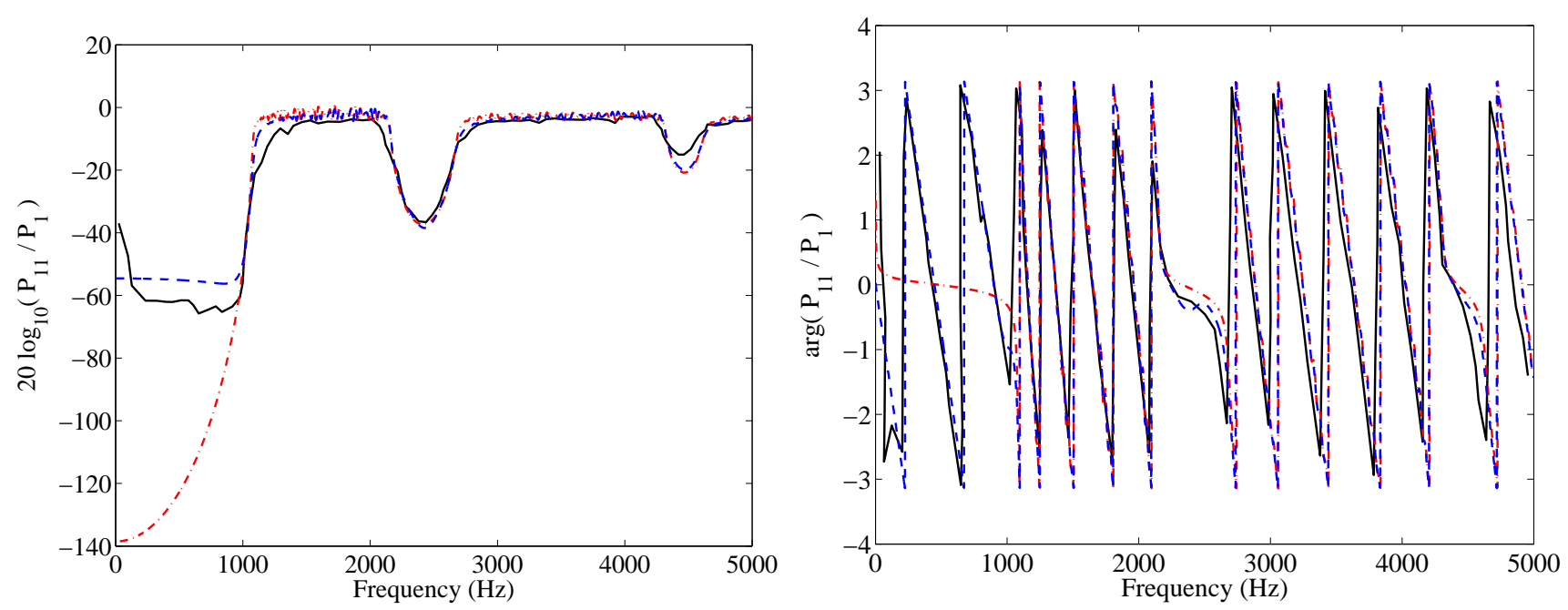

Figure 4: Modulus and argument of the transfer function between the internal pressure at hole 11 and 1: experimental results (black solid), theoretical calculation without external interaction (TMM, red dash-dotted) and with interaction (TMMI, blue dashed).

that is, the propagation of sound between toneholes is not planar, as assumed in the proposed method. This phenomena is one likely cause of the remaining discrepancies. Another is that the model of the mutual interaction assumes that each tonehole is a monopole. In spite of those simplifications, the proposed method gives improved results. Most of the discrepancies between the classical TMM and the measurements are explained by the presence of external tonehole interactions.

Generally speaking, Fig. 5 exhibits a major feature of pass bands: external interaction yields a significant reduction of oscillations with frequency, i.e. a reduction of the standing wave amplitude. This feature was stated by Kergomard [16]. In Appendix B, a theoretical justification is given, allowing the following interpretation:

- without interaction, there is reflection at the end, with standing waves inside the lattice;

- without interaction, standing waves imply the existence of extrema of flow rate, the different holes radiating at different levels;

- the holes radiating strongly have an important influence on the holes radiating weakly, thus there is a kind of equalization of radiation by the different holes, thus a diminution of the apparent standing wave ratio (SWR);

- finally at the input of the lattice there is a diminution of the reflection coefficient.

A consequence is the reduced height of the impedance peaks above the cutoff frequency and a reduction in the radiation directivity lobes in the backward direction [16]. An analysis of the clarinet cutoff frequencies taking into account the external interaction can be found in [22]. 

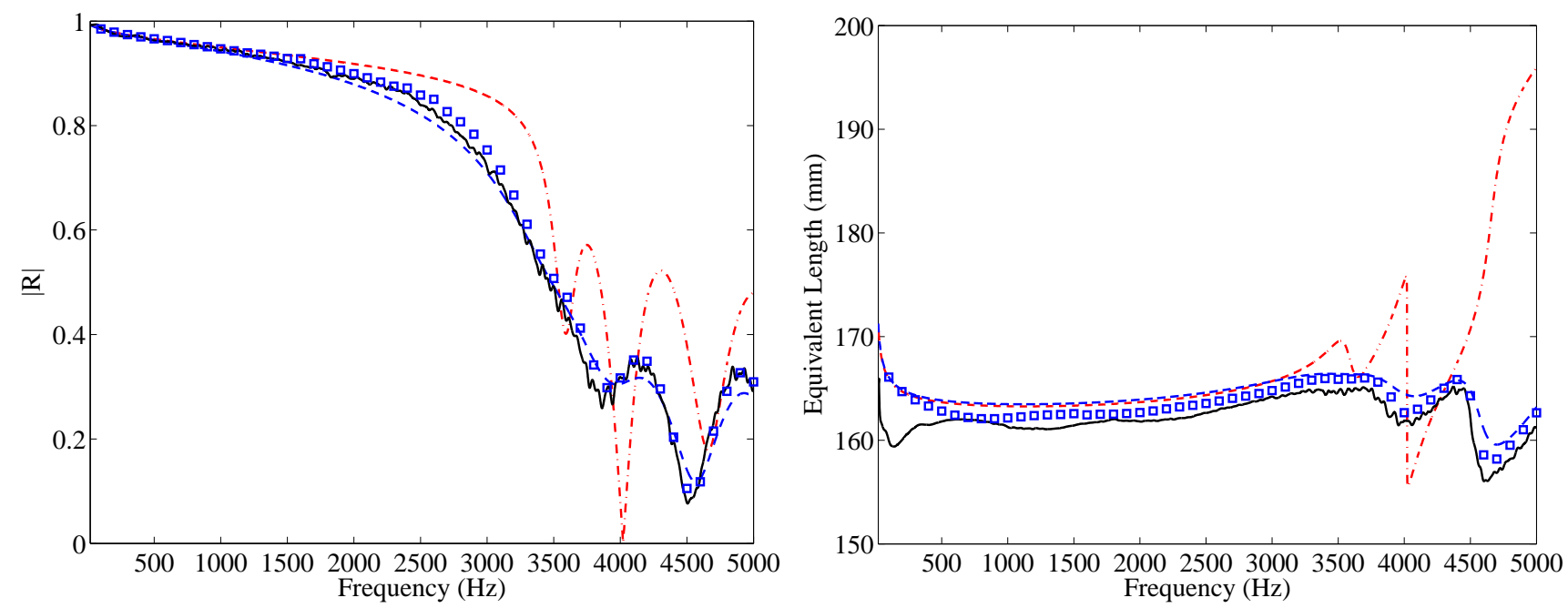

Figure 5: Magnitude of the reflectance (left) and equivalent length (right) of a pipe with 10 toneholes: experimental results (black solid), FEM simulation results (squares), calculations with external interactions (TMMI, blue dashed) and calculations without interactions (TMM, red dash-dotted).

\section{Results for a Saxophone and a Clarinet}

A precise computational FEM model of a complete music instrument is difficult to create and requires significant computation time to solve. Thus, the TMMI model can provide a faster and easier numerical technique which provides satisfactory results for real instruments with complicated geometry, despite the fact that the theoretical description of the toneholes with key pads is overly simplified. In general, we can at least expect that qualitative effects are well represented. Results of the TMM, TMMI, and measurements for an alto saxophone and a clarinet are presented in this section. The saxophone and clarinet were measured with their mouthpieces removed.

\subsection{Input impedance measurements}

The input impedance measurements were made with a multi-microphone system, as described in [19]. A JBL 2426 horn driver is attached to one end of the probe and six PCB Piezotronics condenser microphones (model 377B10) with preamplifiers (model 426B03) are mounted flush with the inner probe wall at $30 \mathrm{~mm}, 60 \mathrm{~mm}, 100 \mathrm{~mm}, 150 \mathrm{~mm}, 210 \mathrm{~mm}$, and $330 \mathrm{~mm}$ from the input plane of the pipe. The microphones are connected to a PCB Piezotronics signal conditioner (model 483C30) and then to a computer through an RME Fireface 800 audio interface. The signals are sampled at $48 \mathrm{kHz}$. The system is excited with a repeated logarithmically-swept sine tone of length 32768 samples and the resulting responses to this signal are averaged in the time-domain, with the first response being discarded. The spectral analysis is performed with an FFT size of 32768, giving a frequency resolution of $1.46 \mathrm{~Hz}$. The pressure spectra at each microphone are used to solve for the forward and backward traveling waves in the system [11], effectively measuring the reflectance of an attached object. The probe is calibrated with three non-resonant loads, as described in [8], though a time-windowing technique [15] is used for the quasi-infinite length pipe. 

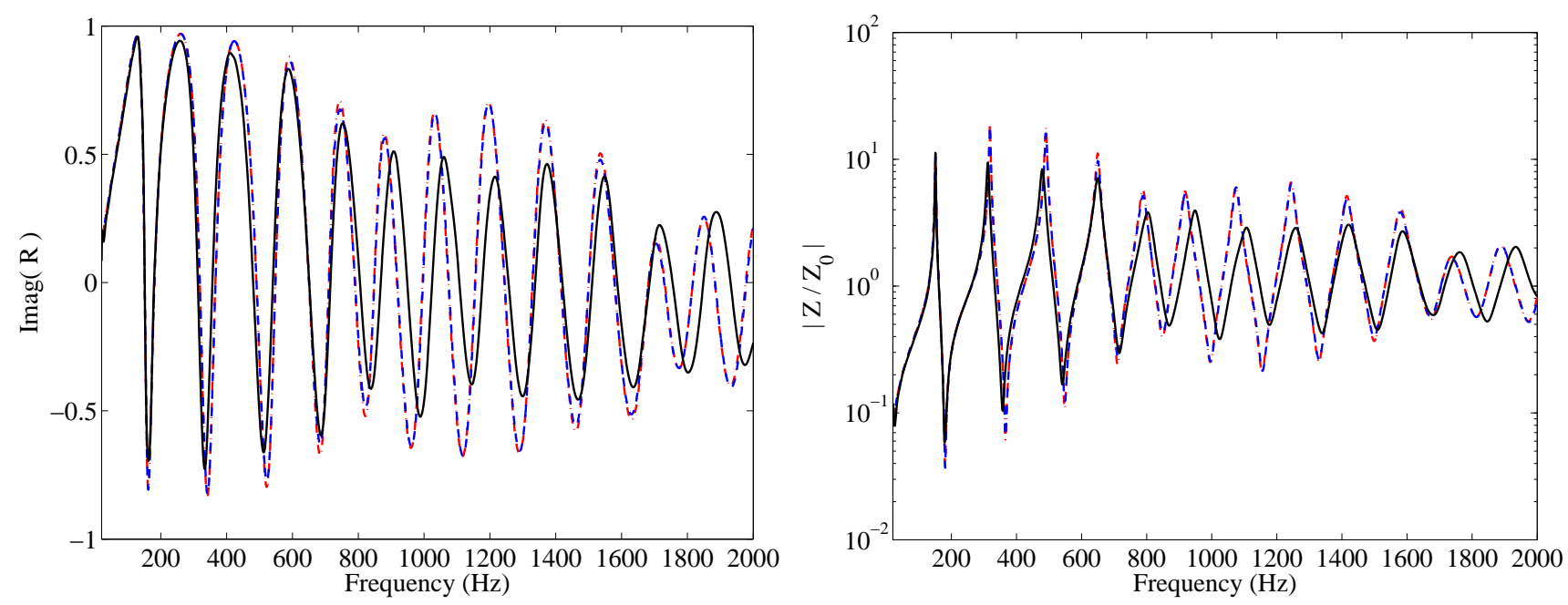

Figure 6: Imaginary part of the reflectance (left) and magnitude of the impedance (right) of an alto saxophone with a low $B$ fingering: experimental results (black solid) calculations with external interactions (blue dashed) and calculations with the TMM (red dash-dotted). Because of there are few open holes, the interaction effects are very small and the two curves TMM and TMMI are barely distinguishable.

A cylindrical pipe of $60 \mathrm{~cm}$ was measured with this system and compared to measurements using a CTTM impedance probe [17], as well as the TMM. All results were within $2 \mathrm{~dB}$ and $1 \%$ frequency accuracy at impedance magnitude extrema between $50-2000 \mathrm{~Hz}$ (the frequency range of interest in the following sections).

\subsection{Saxophone}

The input impedance of an alto saxophone was measured and compared with calculations using the TMMI and classical TMM methods. The instrument is a Selmer Super Action Series II, serial \#438024. The imaginary part of the reflectance and the magnitude of the impedance for the first register written $B_{3}, B b_{4}$, and $C \#_{5}$ fingerings (respectively 146,277 and $330 \mathrm{~Hz}$ ) are shown in Figs. 6 7and 8 . These three fingerings correspond to having a single open tonehole near the bell, a cross-fingering with several holes closed between open holes, and most holes open, respectively. Discrepancies between the experimental data and the calculations in Fig.6for frequencies above about $600 \mathrm{~Hz}$ are likely due to inaccuracies of the bell geometry and model.

As expected, the TMM and TMMI results are nearly identical when few holes are open (Fig.6). As more holes are opened, variations are more apparent and the TMMI tends more toward the experimental results. The magnitude of the impedance peaks are generally reduced by the external interactions. Though difficult to discern in the figures, the resonance frequencies are slighly lower in the TMMI results compared to the TMM.

\subsection{Clarinet}

The input impedance of a $B b$ clarinet was measured and compared with calculations using the TMMI and classical TMM methods. The instrument is a Selmer USA Signet 100, serial \#211240. The imaginary part of the reflectance and the magnitude 

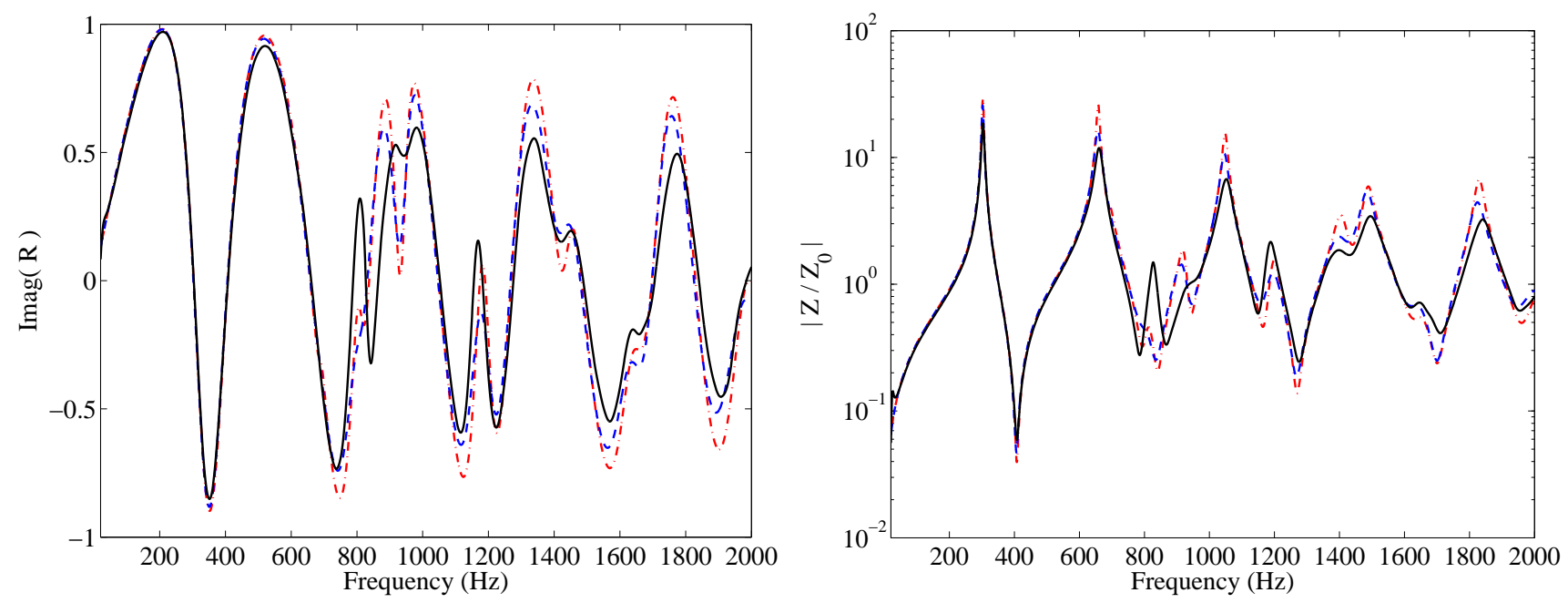

Figure 7: Imaginary part of the reflectance (left) and magnitude of the impedance (right) of an alto saxophone with a $B b$ cross fingering: experimental results (black solid) calculations with external interactions (blue dashed) and calculations with the TMM (red dash-dotted).

of the impedance for the first register written $F_{3}, E b_{4}$, and $G_{4}$ fingerings (respectively 156,277 and $349 \mathrm{~Hz}$ ) are shown in Figs. 9, 10 and 11 As with the saxophone, these three fingerings correspond to having a single open tonehole near the bell, a cross-fingering with several holes closed between open holes, and most holes open, respectively.

Similarly to the case of the saxophone, the resonance frequencies of the clarinet are predicted to be lower when external interactions are accounted for. For fingerings where many toneholes are open, the lowering is on the order of 5-10 cents, which is slightly larger than for the saxophone. As expected, the lowest notes of the instrument, where only a few toneholes are open, are not much affected.

For higher frequencies, the behavior of the instrument changes more significantly. As an example, Fig. 11 displays the imaginary part of the reflectance and the magnitude of the impedance for the fingering $G_{4}(349 \mathrm{~Hz})$ (no fingers down). For the first two resonances, the external interaction only slightly shifts the frequencies to a lower value and the maxima of the impedance corresponds with the zeros of the imaginary part of the reflectance. Between 1600-2000 Hz, however, the impedance magnitude extrema are clearly attenuated by the external interactions and the resonance frequencies more visibly shifted lower.

\section{Conclusion}

The TMMI method provides a more accurate means for the calculation of the acoustics properties of woodwind instrument than the classical TMM, because it accounts for external interactions. As explained in Appendix B, it is not possible to use a simple perturbation of the TMM, but the implementation of the TMMI is rather easy, and the computation time is short. The discrepancies between the TMMI and FEM are rather small and can probably be explained by several factors: first, the values of the radiation-matrix elements are roughly approximated; second, when several adjacent toneholes are closed, further 

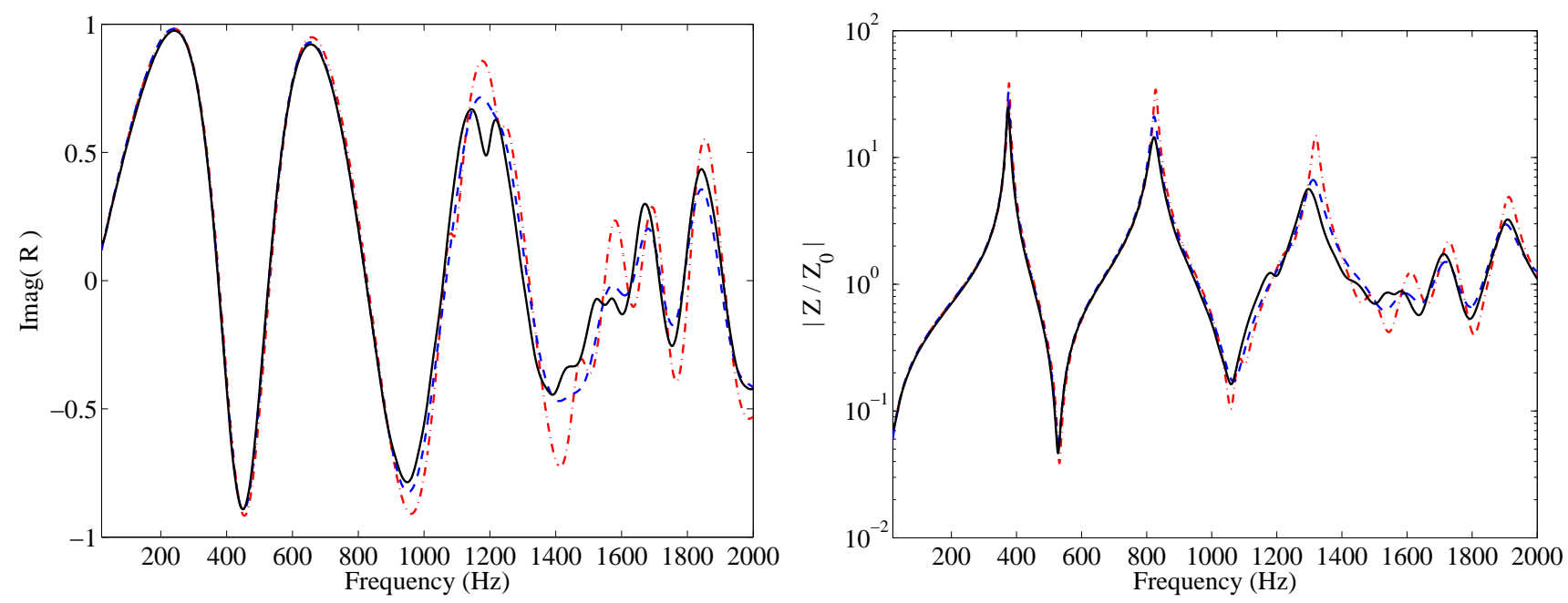

Figure 8: Imaginary part of the reflectance (left) and magnitude of the impedance (right) of an alto saxophone with a $C \sharp$ fingering: experimental results (black solid) calculations with external interactions (blue dashed) and calculations with the TMM (red dash-dotted).

improvement of the higher frequency modeling of a woodwind instrument would require internal coupling of higher-order modes to be accounted for, at least for the determination of the resonance frequencies (notice that these effects do not yield radiation effects, thus dissipative effects, in comparison to the external interactions).

Future work needs to be done for a systematic comparison between theory and experiment for the case of woodwind instruments. This is a long and delicate task because it requires very precise geometrical measurements, including bends and positions of the keys over the tone holes, as well as a precise theory.

Finally, we can summarize some effects of the interactions between holes as:

- The effect of tonehole interactions is generally more important when the toneholes are closer together.

- The order of magnitude of the interaction effect seems to be of the same order for saxophones and clarinets.

- At low frequencies, the effect of interaction is of the order of magnitude of 10 cents when several holes are open, i.e. more than the threshold interval that the ear can perceive (about 4 cents). This is not negligible for instrument-making purpose.

- At higher frequencies, the high-pass filtering behavior of the tonehole lattice allows more flow past the first tonehole and increases the effect of interactions, particularly near and above the cutoff frequency.

- Near and above cutoff, the standing wave ratio is reduced by the effect of interactions. Therefore the effect is important mainly at higher frequencies, and needs to be taken into account for sound simulation or synthesis purposes. 

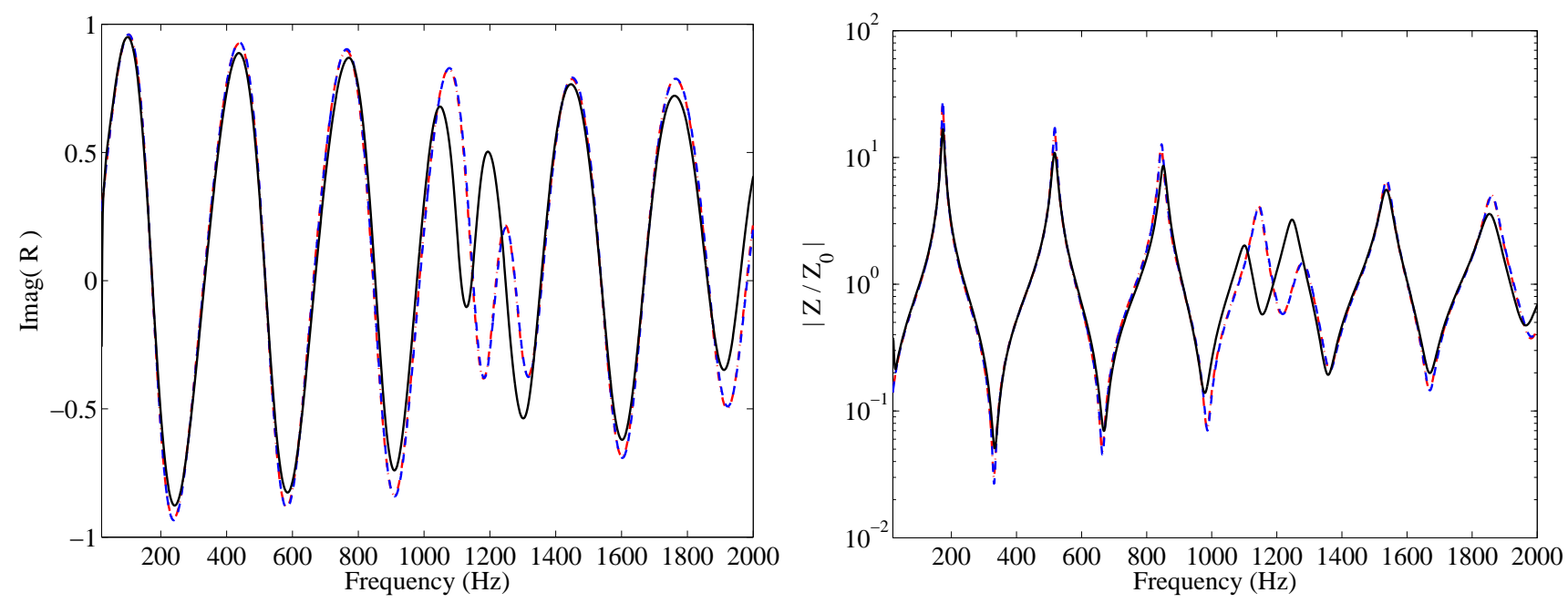

Figure 9: Imaginary part of the reflectance (left) and magnitude of the impedance (right) of a clarinet with a low $F$ fingering: experimental results (black solid) calculations with external interactions (blue dashed) and calculations with the TMM (red dash-dotted). Because of there are few open holes, the interaction effects are very small and the two curves TMM and TMMI are barely distinguishable.

\section{Acknowledgements}

This project was funded in part by a Discovery Accelerator Supplements grant from the Natural Sciences and Engineering Research Council of Canada. The authors also wish to acknowledge the contribution of experimental data by Helmhut Springer during an internship in Le Mans, France in 1986.

\section{References}

[1] Alvin Bayliss, Max Gunzburger, and Eli Turkel. Boundary conditions for the numerical solution of elliptic equations in exterior regions. SIAM Journal on Applied Mathematics, 42:430-451, 1982.

[2] R. Bossart, N. Joly, and M. Bruneau. Hybrid numerical and analytical solutions for acoustic boundary problems in thermo-viscous fluids. J. Sound Vib., 263:69-84, 2003.

[3] René Caussé, Jean Kergomard, and Xavier Lurton. Input impedance of brass musical instruments - Comparison between experimental and numerical models. J. Acoust. Soc. Am., 75:241-254, 1984.

[4] Antoine Chaigne and Jean Kergomard. Acoustique des instruments de musique. Éditions Belin, Paris, France, 2008.

[5] L. Cremer. On the acoustic boundary layer outside a rigid wall. Arch. Elektr. Uebertr, 2:235, 1948.

[6] Jean-Pierre Dalmont and Cornelis J. Nederveen. Radiation impedance of tubes with different flanges: numerical and experimental investigations. J. Sound Vib., 244:505-534, 2001. 

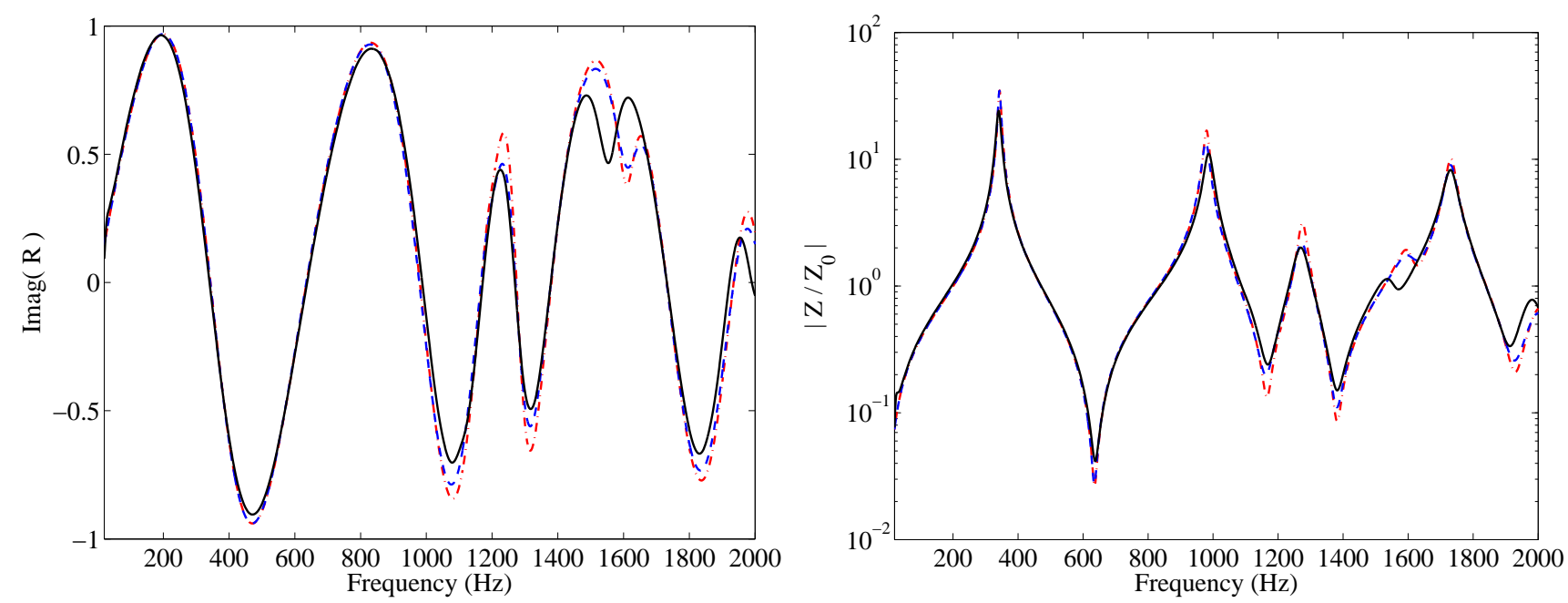

Figure 10: Imaginary part of the reflectance (left) and magnitude of the impedance (right) of a clarinet with an $E b$ cross fingering: experimental results (black solid) calculations with external interactions (blue dashed) and calculations with the TMM (red dash-dotted).

[7] Jean-Pierre Dalmont, Cornelis J. Nederveen, Veronique Dubos, Sbastien Ollivier, Vincent Meserette, and Edwin te Sligte. Experimental determination of the equivalent circuit of an open side hole: Linear and non linear behaviour. Acustica, 88: $567-575,2002$.

[8] P. Dickens, J. Smith, and J. Wolfe. Improved precision in measurements of acoustic impedance spectra using resonancefree calibration loads and controlled error distribution. J. Acoust. Soc. Am., 121(3):1471-1481, 2007.

[9] Vincent Dubos, Jean Kergomard, A. Khettabi, Jean-Pierre Dalmont, Douglas H. Keefe, and Cornelis Johannes Nederveen. Theory of sound propagation in a duct with a branched tube using modal decomposition. Acustica united with Acta Acustica, 85:153-169, 1999.

[10] Dan Givoli and Beny Neta. High-order non-reflecting boundary scheme for time-dependent waves. Journal of Computational Physics, 186:24-46, 2003.

[11] S. Jang and J-G. Ih. On the multiple microphone method for measuring in-duct acoustic properties in the presence of mean flow. J. Acoust. Soc. Am., 103(3):1520-1526, 1998.

[12] W.R. Kampinga, Y.H. Wijnant, and A. de Boer. Performance of several viscothermal acoustic finite elements. Acustica, $96: 115-124,2010$.

[13] Douglas H. Keefe. Acoustic streaming, dimensional analysis of nonlinearities, and tone hole mutual interactions in woodwinds. J. Acoust. Soc. Am., 73:1804-1820, 1983.

[14] Douglas H. Keefe. Woodwind air column models. J. Acoust. Soc. Am., 88:35-51, 1990. 

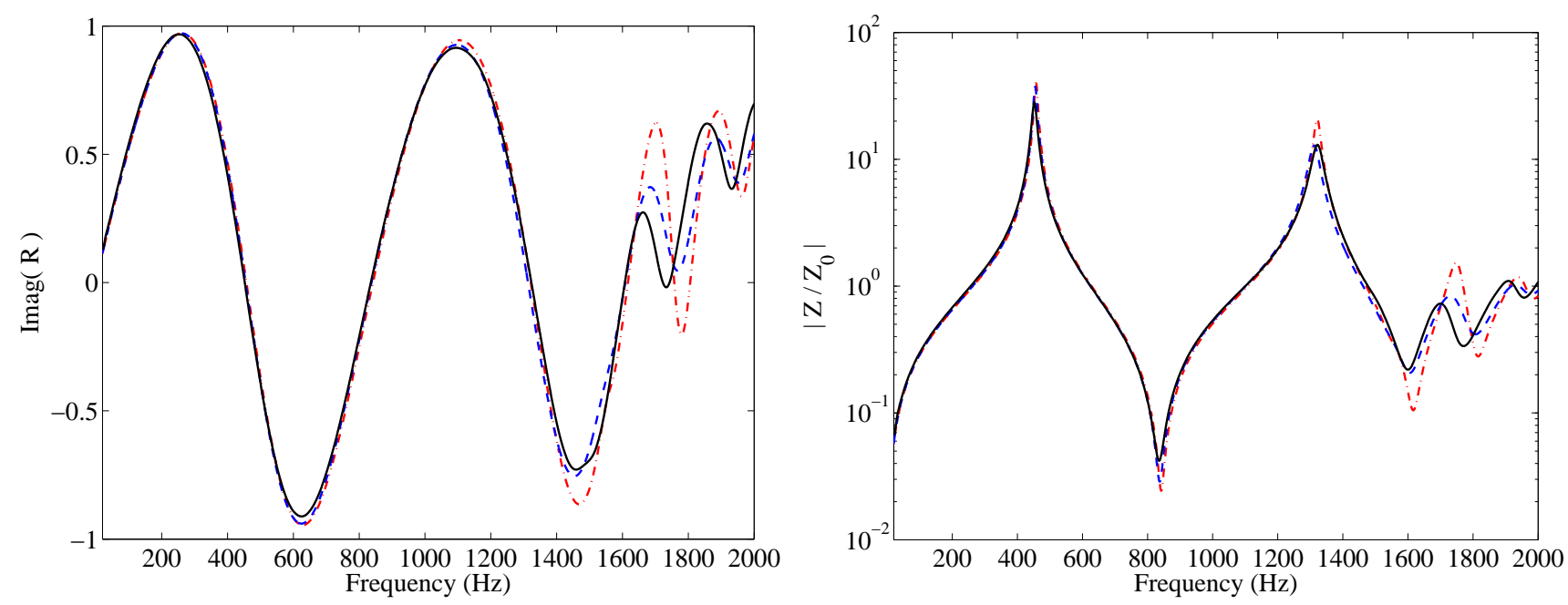

Figure 11: Imaginary part of the reflectance (left) and magnitude of the impedance (right) of a clarinet with a $G$ fingering: experimental results (black solid) calculations with external interactions (blue dashed) and calculations with the TMM (red dash-dotted).

[15] J. Kemp, M. van Walstijn, D.M. Campbell, J. Chick, and R. Smith. Time domain wave separation using multiple microphones. J. Acoust. Soc. Am., 128(1):195-205, 2010.

[16] Jean Kergomard. Tone hole external interactions in woodwind musical instruments. In Proceedings of the 1989 Congress on Acoustics, 1989.

[17] J.C. Le Roux and J.P. Dalmont. A new impedance sensor. In Proceedings of the Acoustics '08 Conference, Paris, France, 2008.

[18] Antoine Lefebvre. Computational Acoustic Methods for the Design of Woodwind Instruments. Ph.D. thesis, McGill University, 2010.

[19] Antoine Lefebvre and Gary P. Scavone. A comparison of saxophone impedances and their playing behavior. In Proceedings of the 2011 Forum Acusticum Conference, Aalborg, Denmark, pages 539-544, 2011.

[20] Antoine Lefebvre and Gary P. Scavone. Characterization of woodwind instrument toneholes with the finite element method. J. Acoust. Soc. Am., 131:3153-3163, 2012.

[21] F.G. Leppington. On the theory of woodwind finger holes. J. Sound Vib., 83:521-532, 1982.

[22] E. Moers and J. Kergomard. On the cutoff frequency of clarinet-like instruments: Geometrical versus acoustical regularity. Acustica, 97:984-996, 2011.

[23] Morse and Ingard. Theoretical Acoustics. Princeton University Press, Princeton, New Jersey, 1968. 
[24] C. J. Nederveen, J. K. M. Jansen, and R. R. van Hassel. Corrections for woodwind Tone-Hole calculations. Acustica united with Acta Acustica, 84:957-966, 1998.

[25] George R. Plitnik and William J. Strong. Numerical method for calculating input impedances of the oboe. J. Acoust. Soc. Am., 65:816-825, 1979.

[26] R. L. Pritchard. Mutual acoustic impedance between radiators in an infinite rigid plane. J. Acoust. Soc. Am., 32(6): $730-737,1960$.

[27] Helmhut Springer. Effet de l'interaction extérieure d'un réseau régulier de trous latéraux percés sur un tuyau sonore. Technical report, Université du Maine, Laboratoire d'Acoustique, Le Mans, November 1986.

[28] S. V. Tsynkov. Numerical solution of problems on unbounded domains. a review. Applied Numerical Mathematics, 27: $465-532,1998$.

\section{Appendix A: General modeling of an open tonehole}

A general model for an open tonehole can be found in Refs. [9, 7]. It includes (negative) inertances in series, which can be concatenated with the transfer matrix of the main tube (Eq. (2)), and a shunt impedance, which can be written as:

$$
Z_{s}=j Z_{0 h}\left\{k t_{i}+\tan \left[k\left(t+t_{m}+t_{r}\right)\right]\right\}
$$

where $Z_{0 h}$ is the characteristic impedance of the tonehole. This includes the effect of the internal added mass, proportional to $t_{i}$, the effect of propagation of the planar mode over the length of the hole chimney $t$, with length corrections corresponding to a matching volume $t_{m}$ and to the radiation $t_{r}$, where:

$$
t_{r}=\arctan \left(Z_{R} /\left(j Z_{0 h}\right)\right) / k
$$

$Z_{R}$ is the radiation impedance. In order to generalized this model to account for external interactions, it is necessary to distinguish the acoustics quantities at the input of a hole (without index) and at its output (index rad), and to write a transfer matrix:

$$
\left(\begin{array}{c}
p \\
u
\end{array}\right)=\left(\begin{array}{cc}
c_{t}-k t_{i} s_{t} & j Z_{0 h}\left(s_{t}+k t_{i} c_{t}\right) \\
Z_{0 h}^{-1} j s_{t} & c_{t}
\end{array}\right)\left(\begin{array}{c}
p \\
u
\end{array}\right)^{r a d}
$$


where $c_{t}=\cos \left[k\left(t+t_{m}\right)\right], s_{t}=\sin k\left(t+t_{m}\right)$. This matrix can be written for each hole, and allows the following matrix relationship to be defined:

$$
\left(\begin{array}{l}
\mathbf{P} \\
\mathbf{U}
\end{array}\right)=\left(\begin{array}{ll}
\mathbb{A} & \mathbb{B} \\
\mathbb{C} & \mathbb{D}
\end{array}\right)\left(\begin{array}{l}
\mathbf{P} \\
\mathbf{U}
\end{array}\right)^{r a d}
$$

The equation $\mathbf{U}^{s}=\mathbf{U}+\mathbb{Y} \mathbf{P}$, obtained from Eqs. (13) and (12), can be written as follows:

$$
\mathbf{U}^{s}=(\mathbb{C}+\mathbb{Y} \mathbb{A}) \mathbf{P}^{r a d}+(\mathbb{D}+\mathbb{Y} \mathbb{B}) \mathbf{U}^{r a d}
$$

If the total length of the tonehole is assumed to be shorter than the wavelength, $\mathbb{A}=\mathbb{D} \simeq \mathbb{I}, \mathbb{C}=\mathbf{0}$, and $B_{n n}=j Z_{0 h} k(t+$ $\left.t_{m}+t_{i}\right)$. Thus, using Eq. (8), Eq. (29) leads to:

$$
\mathbf{U}^{s}=([\mathbb{I}+\mathbb{Y}(\mathbb{Z}+\mathbb{B})] \mathbf{U}
$$

\section{Appendix B: Is it Possible to Compute the External Interaction by the Transfer Matrix Method?}

We consider the equation to be solved:

$$
\mathbf{U}=\left(\mathbf{1}+\mathbf{Y Z}_{R}\right)^{-1} \mathbf{U}_{s}
$$

It is interesting to study if it is possible to solve this equation by perturbation, starting from the TMM method. A quite natural way to do this is to consider that the effect of the external interaction is weak, and to keep a calculation based upon transfer matrices. A first calculation is done without interaction, then the pressures are modified by calculating them with interactions taken into account. The perturbation calculation can be stopped here, but it is possible to iterate it: a new self-impedance is calculated as the ratio of the modified pressure to the unmodified flow rate, then the new flow rates can be calculated again from the transfer matrix method with the modified values of the self-impedances. In practice, the iteration scheme is found to converge for almost all frequencies except low ones. This result is intuitive because in the stop band, the external sound pressure decreases proportionally to the inverse of the distance, while the internal pressure decreases exponentially, therefore the external interaction is more significant.

It is possible to derive a criterion of convergence for the iteration procedure and, when it converges, it is possible to prove that the result is correct. This is done hereafter. At each step $n$ of the calculation, the transfer matrix method leads to the following relationship between the source $\mathbf{U}_{s}$, having a single non-zero element, $U_{s}(1)$, and the pressure and flow rate vectors, $\mathbf{P}^{(n)}$ and $\mathbf{U}^{(n)}$ :

$$
\mathbf{U}_{s}=\mathbf{U}^{(n)}+\mathbf{Y} \mathbf{P}^{(n)}
$$


The calculation is done by defining a diagonal matrix for the termination impedance of each hole (both the direct method and the transfer matrix method can be used):

$$
\mathbf{P}^{(n)}=\mathbf{D}^{(n)} \mathbf{U}^{(n)}
$$

From the knowledge of the flow rate $\mathbf{U}^{(n)}$, the next value of the pressure $\mathbf{P}^{(n+1)}$ is deduced:

$$
\mathbf{P}^{(n+1)}=\mathbf{Z}_{R} \mathbf{U}^{(n)} .
$$

The iteration equation is therefore found to be, with $\mathbf{M}=\mathbf{Y Z}_{R}$ :

$$
\mathbf{U}^{(n+1)}=\mathbf{U}_{s}-\mathbf{M U}^{(n)} .
$$

The recurrence relationship leads to the following solution:

$$
\mathbf{U}^{(n)}=\left[\sum_{i=0}^{n-1}(-1)^{i} \mathbf{M}^{i}\right] \mathbf{U}_{s}+(-1)^{n} \mathbf{M}^{n} \mathbf{U}^{(0)} .
$$

If the norm of the matrix $\mathbf{M}$ is less than unity, the recurrence converges to the solution (31), the series corresponding to a Neumann series expansion.

Now the starting point can be discussed. The first idea is to deduce the solution without interaction from the transfer matrix product :

$$
\mathbf{U}^{(0)}=(\mathbf{1}+\mathbf{Y D})^{-1} \mathbf{U}_{s},
$$

where $\mathbf{D}$ is the diagonal matrix of the self-impedances of $\mathbf{Z}_{R}$. Another possibility is to start with $\mathbf{U}^{(0)}=\mathbf{U}_{s}$ : this implies that the first pressure vector is built with the pressures created by a flow rate located at the first open hole. It can be concluded that the transfer matrix method can be used when the norm of the matrix $\mathbf{Y Z}_{R}$ is less than unity. Because it can be verified that this is not true in the stop band, the perturbation method unfortunately cannot be used in general. This confirms the intuition: looking at Fig. 5] it appears that the effect of external interaction can be very large in stop bands for holes very far apart from each other and the perturbation method cannot converge.

Nevertheless, in pass bands we observe that convergence occurs rapidly when starting from Eq. 377, and even the first order, corresponding to a single perturbation step, is satisfactory. This observation thus justifies the reasoning given in Sec. 3 . 